
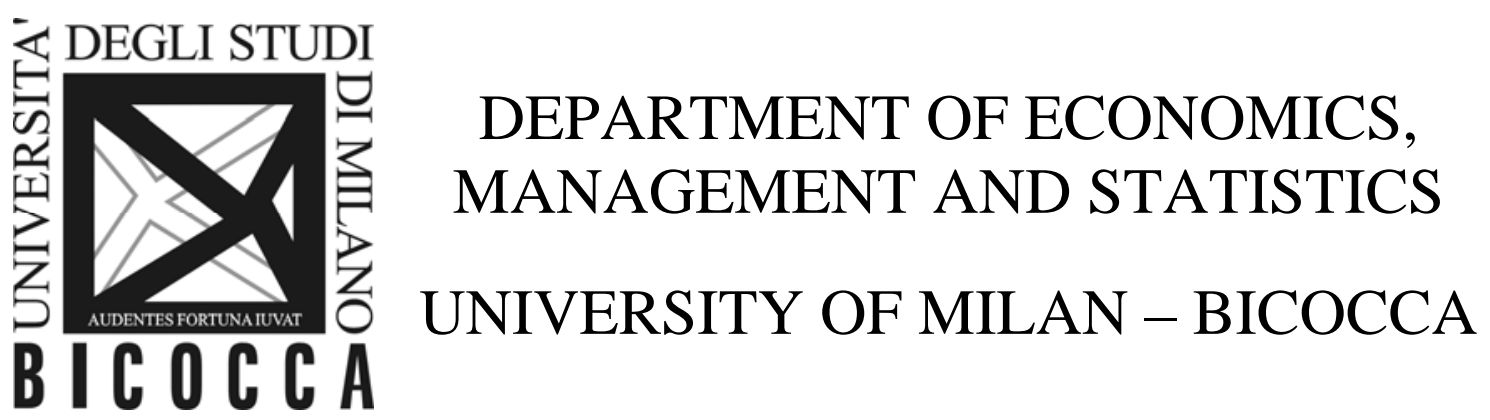

DEMS WORKING PAPER SERIES

\title{
Boundedly Rational Opinion Dynamics in Directed Social Networks: Theory and Experimental Evidence
}

\author{
Pietro Battiston, Luca Stanca
}

No. 267 - January 2014

Dipartimento di Economia, Metodi Quantitativi e Strategie di Impresa Università degli Studi di Milano - Bicocca

http://dems.unimib.it/ 


\title{
Boundedly Rational Opinion Dynamics in Directed Social Networks: Theory and Experimental Evidence
}

\author{
Pietro Battiston* and Luca Stanca ${ }^{\dagger}$
}

January 27, 2014

\begin{abstract}
This paper investigates opinion dynamics and social influence in directed communication networks. We study the properties of a generalized boundedly rational model of opinion formation in which individuals aggregate the information they receive by using weights that are a function of their neighbors' indegree. We then present an experiment designed to test the predictions of the model. We find that both Bayesian updating and boundedly rational updating à la DeMarzo et al. (2003) are rejected by the data. Consistent with our theoretical predictions, the social influence of an agent is positively and significantly affected by the number of individuals she listens to. When forming their opinions, agents do take into account the structure of the communication network, although in a sub-optimal way.
\end{abstract}

Keywords: Social Networks, Learning, Social Influence, Bounded Rationality.

JEL Classification: D85, D83, A14, L14, Z13.

*Department of Economics, University of Milan Bicocca. Piazza dell'Ateneo Nuovo 1, 20126 Milan, Italy. E-mail: me@pietrobattiston.it

$\dagger$ Corresponding author. Department of Economics, University of Milan Bicocca, Piazza dell'Ateneo Nuovo 1, 20126 Milan, Italy. E-mail: luca.stanca@unimib.it 


\section{Introduction}

Research on opinion dynamics and learning in social networks has recently received increasing attention in the economic literature (see Jackson and Yariv, 2010, and Acemoglu and Ozdaglar, 2011, for comprehensive reviews). This growing interest reflected two main factors. At the theoretical level, the development of powerful new tools of analysis. At the empirical level, a significant increase in the availability of data sets to test the theoretical predictions. Several frameworks have therefore been proposed to model opinion dynamics in social networks, based on different assumptions regarding the information transmission mechanisms and the sophistication of individuals. Two main streams can be identified within this literature.

A first group of works includes those that assume perfect rationality, usually together with perfect information about the network structure and the probability distributions of states of nature. The aim of these studies is to establish the optimal strategies and the feasibility conditions in order to reach an estimate of some state of the world which is correct, or asymptotically correct, depending on the setting. The basic framework was laid down by Gale and Kariv (2003). Compared to previous works in the field of social learning (such as Bikhchandani et al., 1992, Banerjee, 1992 and Smith and Sørensen, 2000), their main contribution was to analyze the repeated interaction of Bayesian individuals over non-trivial (exogenously given) network structures. Thereafter, this approach was extended in several directions, as in the work of Acemoglu et al. (2011), who consider the asymptotic properties of opinion aggregation over a growing random network. Acemoglu et al. (2010) assume that information flows are also "tagged", i.e., each agent knows the origin of each element of information she receives, and uses such "metainformation" optimally. In their framework, the only obstacle to recovering the hidden state of nature is the fact that communication is costly, and hence individuals may communicate only for a limited amount of time.

The second group of works follows a more pragmatic approach: since reaching a correct consensus is, even when feasible, generally characterized by a high computational complexity and degree of coordination, it is claimed that studies assuming perfect rationality cannot credibly model the way in which humans - and not only humans - process information when communicating in social networks. As a consequence, rather than starting from individual or social objectives and deriving optimal strategies, these works start from reasonably simple protocols for belief updating, and examine to what extent, under what conditions and with what dynamics, models of opinion dynamics lead to plausible beliefs. ${ }^{1}$

\footnotetext{
${ }^{1}$ This perspective is taken not only in studies focusing on opinion dynamics in social networks, but more generally in the literature on social learning (see e.g. Ellison and
} 
The most common framework in this second group of studies is based on the model of opinion aggregation by DeGroot (1974). Although this work does not explicitly refer to a network environment (as do instead French, 1956, and Harary, 1959, in their studies on social power, later generalized by Friedkin and Johnsen, 1990), since all agents can communicate with all other agents, each individual can attribute a given weight to others' opinion, so that the weights implicitly define a network. These weights, which are constant over time, then determine the evolution and possible convergence of opinions. DeMarzo et al. (2003) have taken over this model, with some variations: in their work, an existing network of connections between agents is explicitly assumed, while opinions are defined as point estimates rather than probability distributions. After considering a general model in which weights can, to some extent, change from period to period, they focus on the case in which individuals attribute the same weight to all neighbors. The assumption that agents do not take into account the topology of the network, but rather update their opinions by simply taking an average of their neighbors' opinions with equal weights, leads to opinions that, even in strongly connected networks, are biased towards the initial beliefs of the most influential (i.e., better connected) subjects. In this setting, agents' social influence depends on their positions in the network and, in particular, on their (and their neighbors') outdegree.

Some works in the related field of distributed sensors (e.g. Olfati-Saber and Murray, 2004) characterize the class of network topologies in which unbiased estimation is obtained even with very simple communication protocols. Other authors extend this framework (Jadbabaie et al., 2012, Jadbabaie et al., 2013), allowing for new (private) information to arrive over time, while assuming that agents process their private information in a Bayesian fashion, and characterize the interplay between the added heterogeneity and the topology of the network. Bala and Goyal (1998) combine the social learning mechanism with a "learning by doing" approach, in which individuals obtain information also by observing their own previous outcomes. Buechel et al. (2012), in their extension of the basic DeGroot model, allow for opinions reported by individuals to differ from actal beliefs, where the difference reflects a preference for conformity or counter-conformity. Hegselmann and Krause (2002) add the feature that the structure of the network itself may be altered endogeneously depending on the similarity of opinions. Their work is part of a literature focusing on the effects of the "bounded confidence" phenomenon in terms of non-convergence (see e.g. Hegselmann and Krause, 2005, Dittmer, 2001, Fortunato, 2004), but its novelty lies in the fact that they consider the interplay of such phenomenon with the structure of an underlying social network.

Fudenberg, 1993). 
In motivating the search for a model of opinion aggregation, the first requirement put forward by DeGroot (1974) is that "The process that it describes is intuitively appealing". Given the explicit quest of this stream of literature for credible models, such models were often preferred over Bayesian ones for interpreting observational and experimental data. Banerjee et al. (2013) exploit a natural experiment focusing on network data from small municipalities in rural India. They conclude that the best predictor for the influence of "injection points" is their eigenvector centrality (as predicted by DeMarzo et al., 2003). Möbius et al. (2010) present a field experiment investigating the diffusion of information over the network defined by friendship relations on Facebook, and seed such network artificially with noisy signals on some hidden state of the world. This allows the authors to ascertain the presence of strong information decay, test the main predictions of the DeGroot (1974) model, and compare them with the possibility of tagged information (as proposed by Acemoglu et al., 2010).

In a study closely related to the present one, Corazzini et al. (2012) present a laboratory experiment aimed at testing the presence of persuasion bias in opinion formation within communication networks. Consistent with the predictions of DeMarzo et al. (2003), their results indicate that the social influence of individuals depends on their eigenvector centrality, which in turn depends on the number of other individuals who, directly or indirectly, listen to them (i.e., their outdegree). The findings, however, are also consistent with the presence of an indegree effect, as agents with higher indegree have higher social influence. In order to explain this phenomenon, Corazzini et al. (2012) suggest a framework based on the assumption that the weights each individual places on her neighbors' opinions are positively related to their neighbors' indegree. Intuitively, more informed individuals receive higher weights when opinions are updated and, as a consequence, also have higher social influence.

Against this background, the objective of this paper is twofold. First, we extend the framework by Corazzini et al. (2012) to study a model of opinion formation that provides a simple generalization of DeMarzo et al. (2003). We also derive some results concerning more generally linear updating models, and characterize the way in which efficiency depends on the topology of the underlying network. Second, we present a laboratory experiment explicitly designed to test the causal effect of indegree on social influence. The structure of the directed network used in the experiment allows us to manipulate indegree without affecting the outdegree and eigenvector centrality of different nodes, thus providing a clean test of the effects of indegree on social influence.

We show that, in balanced networks, placing higher weight on neighbors with higher indegree is less efficient than placing equal weights on all 
neighbors. On the other hand, in unbalanced networks it is generally more efficient to place higher weight on neighbors with higher indegree, and there exist networks in which it is optimal to place weight only on agents with highest indegree. Empirically, we find strong evidence of an indegree effect on opinion formation. The social influence of an agent is positively and significantly affected by the number of individuals she listens to.

The remainder of the paper is structured as follows. Section 2 provides the theoretical framework (technical details are in Appendix A). Section 3 describes the experimental design (experimental instructions are in Appendix B). Section 4 presents the results. Section 5 concludes with a discussion of the key findings.

\section{Theoretical Framework}

Following DeMarzo et al. (2003), consider a setting where a set $\mathcal{N}=\{1, \ldots, N\}$ of agents, communicating within a social network, want to estimate some unknown state of the world represented by the parameter $\theta \in \mathbb{R}$. Each agent starts with some initial information $x_{i}$ (henceforth referred to as a signal) about $\theta$. For simplicity, we assume that $x_{i}=\theta+\varepsilon_{i}$, with $\varepsilon_{i} \sim N\left(0, \sigma^{2}\right)$ independent across agents. The structure of the network is represented as a directed graph with adjacency matrix $q$, where $q_{i j}=1$ if agent $i$ listens to agent $j$, and 0 otherwise (we assume $q_{i i}=1$ for every $i$ ). ${ }^{2}$ We denote as $S_{i} \subset \mathcal{N}$ the listening set of an individual $i$, that is, $j \in S_{i} \Longleftrightarrow q_{i j}=1$. Communication takes place in discrete time: at each $t \geq 0$, agents report their current belief to their neighbors. Defining the vector of initial beliefs as $y^{0}=x$, we assume that, for each $t \geq 0$, agent $i$ updates her belief according to an updating rule

$$
y_{i}^{t+1}=f_{i}\left(y_{i_{1}}^{t}, \ldots, y_{i_{K}}^{t}\right)
$$

where $i_{1}, \ldots, i_{K}$ are the agents in $i$ 's listening set $S_{i}$ (notice that $i \in S_{i}$ ). Once the network structure $q$, the updating rules $f_{1}, \ldots, f_{N}$, and the initial signals $x$ are determined, the evolution of opinions is obtained. Given an agent $i$, if $y_{i}^{\infty}=\lim _{t \rightarrow \infty} y_{i}^{t}$ exists, it will be referred to as her convergence or asymptotic belief. In what follows, we will be particularly interested in the case in which convergence beliefs exist and coincide for all $i$ : if this is the case, we will refer to such limit as the consensus belief, and denote it simply as $y^{\infty}$.

\footnotetext{
${ }^{2}$ Notice that in the graphical representations of networks presented below, an arrow from $i$ to $j$ means that agent $i$ talks to (rather than listens to) agent $j$, that is, $q_{j i}=1$. This is different from standard convention, but consistent with the instructions of the experiment presented in Section 3 and with the direction of information flows.
} 
The foundations for this framework were laid by DeGroot (1974), who considers the case in which "opinions" are probability distributions rather than real numbers, and $f$ is linear: ${ }^{3}$

$$
y_{i}^{t+1}=\sum_{j \in S(i)} \pi_{i j} y_{j}^{t}
$$

where $\pi_{i j}$ is the weight placed by agent $i$ on agent $j$. DeMarzo et al. (2003), following the studies on social power of French (1956) and Harary (1959), introduce an exogenously given network structure $q$, and analyze in detail the specific updating rule

$$
y_{i}^{t+1}=\sum_{j \in S(i)} \frac{y_{j}^{t}}{|S(i)|} .
$$

One key feature of the boundedly rational updating rule in (2) is that agents do not use in any way the information contained in $q$ : they simply form their opinion by averaging all opinions they get to know, irrespective of the network structure.

Consider now a generalized boundedly rational updating rule (henceforth GBR) that is still linear, but with weights defined as follows:

$$
\pi_{i j}=\frac{q_{i j} d_{j}^{\rho}}{\sum_{h} q_{i h} d_{h}^{\rho}}
$$

where $d_{j}$ is agent $j$ 's indegree $\left(d_{j}=|S(j)|-1\right)$ and $\rho \in[0, \infty)$ is a fixed parameter. Such rule, which was first introduced by Corazzini et al. (2012), provides a simple generalization of the updating rule in (2), while incorporating plausible and interesting features. Intuitively, when weighting the opinions of neighbors, agents attribute relatively more importance to those neighbors who have more direct sources of information, i.e., neighbors with higher indegree. To illustrate, let us consider some examples.

When $\rho=0$,

$$
\pi_{i j}=\frac{q_{i j}}{\sum_{h} q_{i h}}=\frac{q_{i j}}{|S(i)|}
$$

we obtain the updating rule in (2), as in DeMarzo et al. (2003): agents update their opinions by averaging the opinions they get to know, while placing equal weights on all neighbors.

When $\rho=1$,

$$
y_{i}^{t+1}=\frac{\sum_{j=1}^{n} q_{i j} d_{j} y_{j}^{t}}{\sum_{j=1}^{n} q_{i j} d_{j}}
$$

\footnotetext{
${ }^{3}$ In the context considered by DeGroot (1974), $S(i)$ corresponds to $\{1, \ldots, N\}$.
} 
i.e., the opinion of each neighbor is weighted proportionally to her indegree.

In the limit case $\rho \rightarrow \infty$,

$$
y_{i}^{t+1}=\sum_{j \in \arg \max _{h} q_{h i} d_{h}} \frac{y_{j}^{t}}{\left|\arg \max _{h} q_{h i} d_{h}\right|}
$$

so that each agent only listens to the individual(s) with maximum indegree in her listening set. This limit case is useful to provide an intuition of what happens more generally for high values of $\rho$ : individuals with higher indegree tend to be the most influential. ${ }^{4}$

DeMarzo et al. (2003) show that in a strongly connected network, i.e. where every agent can influence every other agent, any linear updating rule such that $\pi_{i j}>0$ whenever $q_{i j}>0$ guarantees convergence to a consensus belief..$^{5}$ This implies that, in our setting, convergence is ensured for any $\rho \in$ $[0, \infty) .{ }^{6}$ Moreover, if we rewrite Equation (1) in matrix form as $y^{t+1}=\Pi y^{t}$, the vector of consensus beliefs $\bar{y}^{\infty}$ must satisfy the condition

$$
\bar{y}^{\infty}=\Pi \bar{y}^{\infty} .
$$

i.e. $y^{\infty}$ is a right eigenvector of $\Pi$, with eigenvalue 1 . DeMarzo et al. (2003) also show that $y^{\infty}$ can be written as a weighted sum of the initial signals:

$$
y^{\infty}=\sum_{i=1}^{N} w_{i} x_{i}
$$

with $w$ being the unique (normalized) solution to

$$
w \Pi=w .
$$

If we consider $\Pi$ as the adjacency matrix of a weighted network, Equation (7) can be interpreted as stating that the social influence $w_{i}$ of an individual $i$ corresponds to her eigenvector centrality (Bonacich, 1972, Jackson, 2010). In the case of the updating rule in Equation (2), $w_{i}$ also corresponds, up to a

\footnotetext{
${ }^{4}$ It should be noted, however, that networks can be designed in which, even for arbitrary large $\rho$, agents with maximum indegree and maximum outdegree do not have highest social influence.

${ }^{5}$ This is a particular case of their Theorem 1, exploiting the fact that the listening matrix is row-stochastic, and hence describes a Markov chain which is irreducible and aperiodic.

${ }^{6}$ The result does not apply to the limit case $\rho \rightarrow \infty$ : in fact, with the rule described in Equation (2), convergence of beliefs is not guaranteed, as can be verified from the simple counterexample defined by $S(A)=\{B, D\}, S(B)=\{A\}, S(C)=\{B, D\}, S(D)=\{C\}$. In this case, agents $A$ and $B$, who have maximum indegree and are not directly connected, will never change their own beliefs, and hence, assuming their initial signals differ, agreement will not be reached.
} 
scaling factor, to the Katz centrality of $i$ in the original network (Katz, 1953). It is important to observe that Equation (7) implies that, in the updating rule proposed by DeMarzo et al. (2003), social influence is increasing in outdegree (see Appendix A, Theorem 7).

Let us now consider whether consensus beliefs are correct, in the sense of being optimal aggregates of agents' initial information. Given that all signals are equally informative, the consensus belief is correct if $w_{i}=\frac{1}{N} \forall i$, i.e.

$$
y^{\infty}=\frac{\sum_{i=1}^{N} x_{i}}{N}=\bar{x}
$$

Different updating rules can therefore be compared by using the following measure of efficiency:

$$
E=-\sum_{i=1}^{N}\left(w_{i}-\frac{1}{N}\right)^{2}
$$

where $E=0$ if consensus beliefs are correct, while $E<0$ otherwise.

DeMarzo et al. (2003) have analyzed the GBR rule under the restriction $\rho=0$, showing that it will not, in general, lead to a correct consensus in most network structures, as more connected agents have excessive social influence. When $\rho>0$, the GBR rule does not always neutralize such inefficiency: while it may lead to a consensus which is closer to the correct one than for $\rho=0$, this is not a general rule. We thus start by asking more generally whether there exist rules of thumb that lead to correct beliefs for any given network structure. The answer is provided by the following theorems.

Theorem 1 Given any strongly connected network $\overline{\mathcal{G}}$, there exists a linear updating rule $F_{\overline{\mathcal{G}}}$ which guarantees convergence to the correct consensus.

Proof. See Appendix A.

Theorem 2 Given any linear updating rule $\bar{F}$ with weights $\bar{\pi}_{i j}$ which depend on local properties of the network around $i$, there exists a strongly connected network $\mathcal{G}_{\bar{F}}$ on which $\bar{F}$ does not guarantee convergence to the correct consensus.

Proof. See Appendix A.

While the first result is positive, stating that for any given network it is possible to find an optimal linear rule, the second ends the quest for the "perfect" rule of thumb: linear rules cannot be both correct and simple, 
in the sense that weights are determined only by the local properties of a network. $^{7}$

For a given value of $\rho$, the efficiency of the resulting updating rule will clearly depend on the topology of the network. We will denote as $E_{\rho}(\mathcal{G})$ the efficiency of the GBR rule when implemented over a network $\mathcal{G}$ with the given value of $\rho$, and as $\rho^{*}(\mathcal{G})$ the value of $\rho$ that maximizes $E_{\rho}(\mathcal{G})$. In the following, some relevant classes of networks will be defined on which the GBR rule displays particular features in terms of efficiency. In all cases, it will be assumed that networks are strongly connected.

Let us define two nodes $(j, k)$ as equivalent if, after switching their labels, it is possible to arrange the other labels of the network in order to obtain an exact copy of the original one. Then, we can provide the following definitions:

Definition 1 A network structure is anonymous if all nodes are equivalent. ${ }^{8}$

Definition $2 A$ belief updating rule $F$ is anonymous if all $f_{i}$ are symmetric in arguments $y_{j}^{t}$ and $y_{k}^{t}$, for any pair of equivalent nodes $j, k \neq i$.

In other words, a rule $F$ is anonymous if the labels of agents do not play any role. Those two definitions allow us to state the following basic result, which generalizes Theorems 1 and 2 in French (1956), ${ }^{9}$ as well as Theorem 9 in Harary (1959).

Lemma 1 On any given anonymous, strongly connected network $\mathcal{G}$ on which all agents play a same linear anonymous updating rule,

1. The beliefs in each period are uniquely determined by $\pi_{11}, \ldots, \pi_{N N}$, the self attributed weights,

2. The asymptotic consensus beliefs are correct: $w=\left(\frac{1}{N}, \ldots, \frac{1}{N}\right)$.

\footnotetext{
${ }^{7}$ It is interesting to consider a constructive proof of Theorem 1: an algorithm which for any given network $\overline{\mathcal{G}}$ finds a linear rule which is efficient over it. Such algorithm is described in Example 1 of Appendix A, and is based on the existence of a closed path $p_{N}$ passing through all nodes of the network (possibly more than once). Indeed, it clearly does not qualify as a "rule of thumb": in particular, the choice of $p_{N}$ requires significant ex-ante coordination among agents.

${ }^{8}$ This is stronger than the concept of regular graph, which only considers the indegree of vertices (rather than their position in the network), and corresponds instead to the concept of "automorphic group" employed by Harary (1959).

${ }^{9}$ While Theorem 2 by French (1956) is indeed a special case of Lemma 1, his Theorem 1 additionally states that on complete networks, the consensus belief is reached in one step.
} 
Proof. See Appendix A.

One may wonder if anonymity or linearity alone is a sufficient requirement for Lemma 1 to hold. The answer is negative: two counter-examples are given, respectively, by the rules "weight the opinion coming from the highest labeled neighbor as much as the average of all others" (which is linear but not anonymous) and "weight the highest opinion coming from the neighbors as much as the average of all others" (which is anonymous but not linear).

Theorem 3 The GBR rule will, for any choice of $\rho$, (perform equally, and) converge to the true average of signals on any anonymous, strongly connected network.

The theorem is a mere consequence of the GBR updating rule being linear and anonymous, and of Lemma 1. For an example, see Figure 1, displaying a complete network with four nodes: since the value of $\rho$ is irrelevant, the efficiency of the GBR rule is constant, and the value of the loss function is equal to 0 .

Figure 1: An anonymous complete network
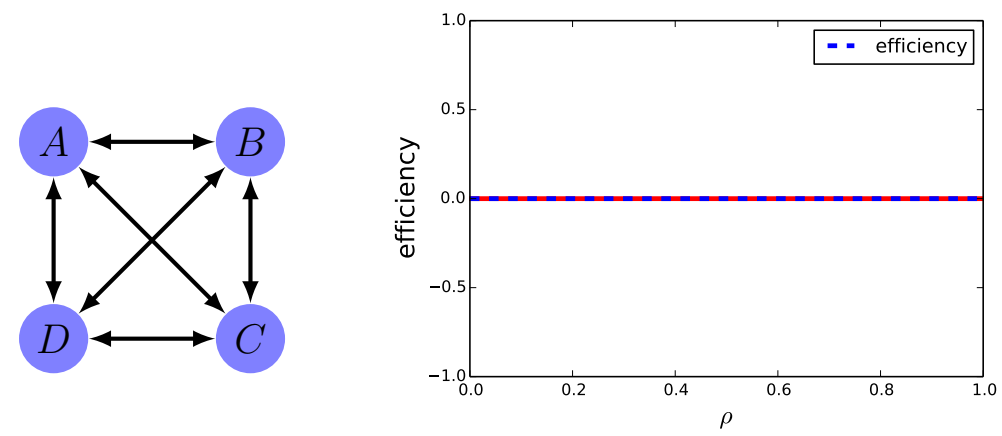

The requirement of anonymity of a network is a very strong one. In fact, the class of networks on which the GBR rule will perform equally for any given $\rho>0$ is significantly larger than that of anonymous ones. To see this, notice that on any regular network, ${ }^{10}$ independently of $\rho$, each agent will attribute the same importance to the opinion of each neighbor, since all of them will have the same indegree. Interestingly, the regularity of the network is also a necessary condition for $\rho$ to be irrelevant, as stated in the following theorem.

Theorem 4 On a strongly connected network $\mathcal{G}$, the convergence belief and social weights obtained under a GBR rule with given $\rho>0$ coincide with the ones obtained under $\rho=0$ if and only if $\mathcal{G}$ is regular.

\footnotetext{
${ }^{10} \mathrm{~A}$ directed network is regular if all nodes have the same indegree and outdegree (the two must necessarily coincide).
} 
Proof. See Appendix A.

The next result concerns a wider class of networks, which includes all anonymous ones, but also many others, such as undirected networks and regular networks.

Definition 3 A network is said to be balanced if each node has indegree equal to outdegree. ${ }^{11}$

Theorem 5 On any given balanced, strongly connected network $\mathcal{G}$, the $G B R$ with $\rho=0$ is more efficient than with any $\rho>0$.

The proof for this theorem is in Appendix A. Still, it can be interesting to consider an intuitive explanation. On this class of networks, the problem of persuasion bias is attenuated. Although it is present in the first period, in the long run agents with higher outdegree exploit their influence to convey richer information, since they also have higher indegree. Intuitively, their belief has higher weight, but their own initial opinion gets diluted in their belief. This does not occur when $\rho>0$, which causes agents with higher outdegree to place even higher weight on their own signal.

For an application of Theorem 5, consider the network structure in Figure 2. While it is not anonymous (nodes $B$ and $D$ are identical, but they differ from nodes $A$ and $C$ ), it shares with the complete network the feature of being undirected. Figure 2 shows that the optimal value of $\rho$ is 0 . Notice that Theorem 5 only holds asymptotically: it is easy to provide counterexamples in finite time, by replacing in the definition of efficiency (Equation 9) the weights $w_{i}$ with those calculated after a finite number of periods.

Figure 2: An undirected, and hence balanced, network
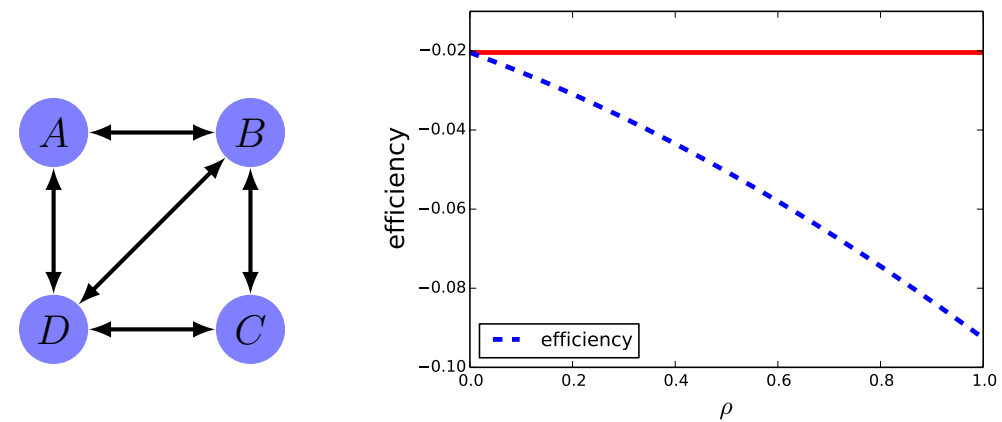

More importantly for our purposes, outside of those specific classes of networks, things change radically. While there can be cases of unbalanced

\footnotetext{
${ }^{11}$ This definition is adopted from Olfati-Saber and Murray (2004). It is more general than the ones used by Corazzini et al. (2012) and DeMarzo et al. (2003).
} 
networks on which a lower $\rho$ still means higher efficiency, this is not the rule. The network presented in Figure 3, which is the one used in the experiment presented in Section 3, is characterized by an optimal value of $\rho$ that is positive (0.04). For higher values of $\rho$, the social influence of $B$, who has a higher indegree, increases, while the one of $D$ decreases.

Figure 3: The network used in the experimental setting
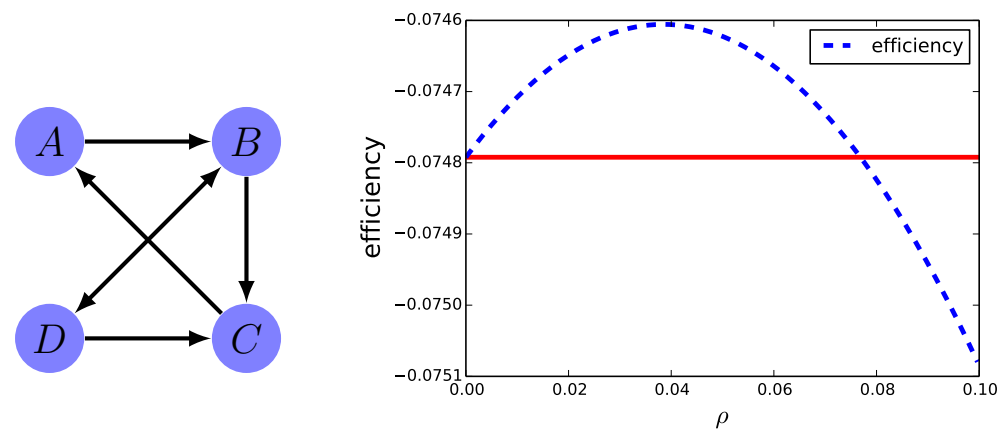

The following theorem states that no value of $\rho$ is in principle "too high".

Theorem 6 Given any $\bar{\rho} \in[0, \infty)$, there exists a strongly connected network $\overline{\mathcal{G}}$ such that $\rho^{*}(\overline{\mathcal{G}})>\bar{\rho}$.

Proof. See Appendix A.

In short, our results can be summarized as follows. In anonymous networks the weights placed on neighbors are irrelevant, since all linear rules are efficient. In balanced networks, placing higher weight on neighbors with higher indegree $(\rho>0)$ is generally less efficient than simply placing equal weights on all neighbors $(\rho=0)$. Finally, in unbalanced networks, it may be optimal to place higher weight on neighbors with higher indegree, and there exist networks in which the optimal value of $\rho$ is arbitrarily high. In the next Section we present an experimental test of the effects of indegree on opinion dynamics and social influence.

\section{Experimental Design}

The experiment is designed to test the effects of agents' position in a communication network on their social influence. More specifically, our experimental design allows us to manipulate agents' indegree without affecting their outdegree and the corresponding eigenvector centrality. Therefore, it enables us to provide a clean test of the effect of indegree on social influence, that would be absent under either Bayesian updating or boundedly rational updating à la DeMarzo et al. (2003). 


\subsection{Task}

At the beginning of the experimental task, individuals are anonymously matched in groups of four. In each group, subjects are connected through a communication network, and each subject is assigned a label $(A, B, C, D)$ that defines her position in the network. Each subject is assigned an integer number randomly drawn from a normal distribution, henceforth referred to as a signal, denoted with $x_{A}, x_{B}, x_{C}, x_{D}$, respectively. The task is based on a discrete time setting over 8 rounds. In each round the subjects are asked to guess the average $\bar{x}=\frac{x_{A}+x_{B}+x_{C}+x_{D}}{4}$ of the four signals in their group. The choice of replacing the population mean $(\theta)$ with the sample mean $(\bar{x})$ is made to simplify the experimental task, as it allows subjects to concentrate on the aggregation of information rather than statistical inference.

In order to be able to update their beliefs, at the beginning of each round subjects receive information from the other group members they are connected to. More specifically, at time $t$ each individual is informed about the guesses at time $t-1$ of the other group members connected to her (the network structure, that defines who receives information from whom, is described in the next subsection). Therefore, while in the first round subjects only directly know their own signal, over successive rounds they directly or indirectly receive information about the signals received by the other group members. If all four group members optimally process the information they receive, over successive rounds each of them can correctly guess $\bar{x}$.

The mechanism for eliciting beliefs is as follows: each individual is informed that at the end of the session, one round will be randomly extracted to determine earnings. Given the guess $y^{*}$ of the individual in that round, and the average of signals in the group $(\bar{x})$, the individual's payoff is 15 euro minus the absolute difference between $y^{*}$ and $\bar{x}$, in addition to a show-up fee of 5 euros. This implies that individuals have an incentive to report in each round their best guess for the group average. We adopt a triangular scoring rule for three reasons. First, a quadratic scoring rule, commonly used for belief elicitation, would substantially complicate the calculation of payoffs, hence increasing the likelihood of mistakes due to mis-comprehensions. Second, for a given average gain, a quadratic scoring rule would increase the likelihood of earning very small payoffs, thus adversely affecting the incentive to exert effort in the task. Third, given normality of signals, our setting is perfectly symmetric, so that the median of the posterior's distribution coincides with the mean. In addition, more generally, experimental subjects were explicitly instructed to update their beliefs by averaging all known and inferred signals, and control questions indicated that such instruction was clearly understood. 


\subsection{Treatments}

Figure 4 describes the strongly connected directed network structure we use in the experiment. The number of nodes is small in order to provide a simple setting for the experimental subjects, but at the same time sufficiently large to imply interesting opinion dynamics. The network structure implies that $A$ is informed about past beliefs of $C, B$ is informed about past beliefs of $A$ and $D, C$ is informed about past beliefs of $B$ and $D$, while $D$ is informed about past beliefs of $B$. The indegree and outdegree of the four nodes are $A=(1,1), B=(2,2), C=(2,1), D=(1,2)$, respectively. The reason for choosing this specific network structure is that, as explained below, it allows us to cleanly test the key hypotheses of the experiment.

Figure 4: Structure of the communication network

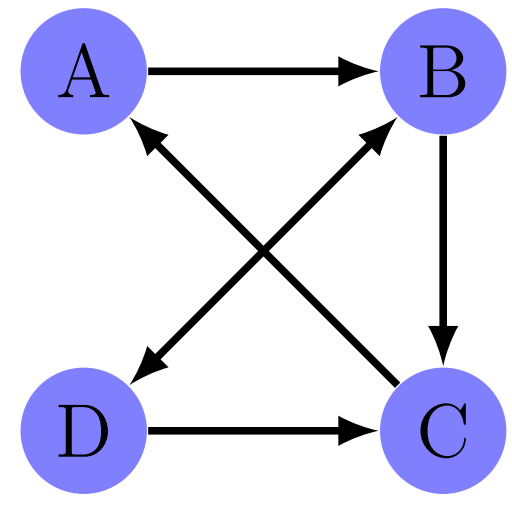

The treatment variable is the node that the subject is assigned to within the network. The four treatments (nodes $A, B, C$ and $D$ ) are implemented in a within-subjects design. This means that, in an experimental session, each subject performs the task four times, thus taking part in four subsequent phases of 8 rounds (32 rounds overall). In each of the four phases, each subject is randomly assigned to a different node (position) in the network. Therefore, within a session, each individual is assigned each node in exactly one of the four phases. Subjects receive a different set of signals at the beginning of each phase, while the composition of the groups is unchanged throughout the four phases.

Since we aim at assessing if and how agents' social influence is affected by their position in the network, it is important to control for the possible confounding effects of the labels attached to each node $(A, B, C, D)$ and of subjects' visual location in the network (upper left, upper right, bottom left, bottom right). It is possible, for instance, that subjects tend to give more importance (higher weight) to nodes denoted by letters that come first in the alphabet (e.g. $A$ vs $D$ ). Similarly, subjects might tend to give more importance to nodes located in the top-left of the network visual display, as 
opposed to the bottom-right. In order to control for such spurious effects, we implemented the four treatments in each of four sessions keeping constant the networks structure, while changing in each session the spatial disposition of the nodes, as detailed in Figure 5. ${ }^{12}$ The same four sets of signals were used in each of the four sessions. Therefore, by implementing the four network nodes with all possible labels and visual locations, while keeping signals constant, we were able to control for any possible confounding effects and cleanly identify the causal effects of network structure.

Figure 5: Network structure, by session
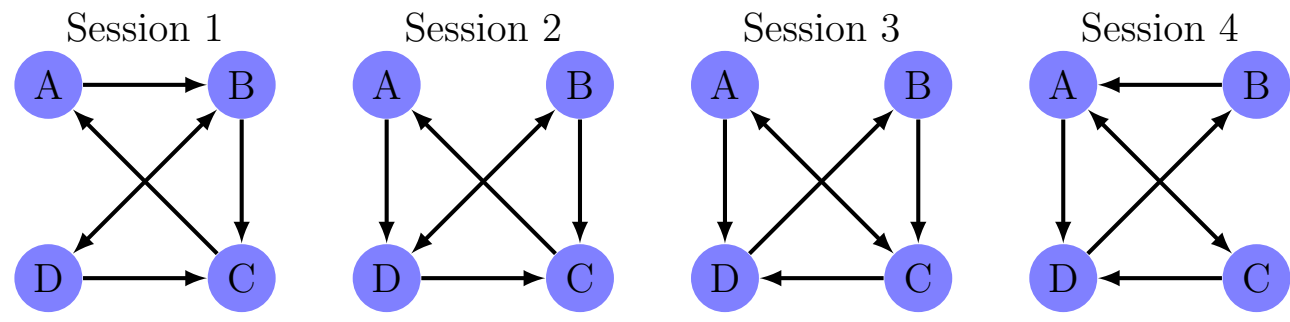

\subsection{Hypotheses}

Consider the network structure in Figure 4. As detailed in Table 1, there exists a set of strategies that allows each of the four network members to find out $\bar{x}$ in just four rounds. Indeed, there exist several possible combinations of strategies that result in correct beliefs. With such optimal strategies, the four agents have equal social influence weights in consensus beliefs, i.e. $w^{*}=[0.25,0.25,0.25,0.25]$.

Table 1: Optimal strategies for each network position, by round

\begin{tabular}{ccccc}
\hline \hline Round & $A$ & $B$ & $C$ & $D$ \\
\hline 1 & $x_{A}$ & $x_{B}$ & $x_{C}$ & $x_{D}$ \\
2 & $\frac{x_{A}+y_{C}^{1}}{2}$ & $\frac{x_{B}+y_{A}^{1}+y_{D}^{1}}{3}$ & $\frac{x_{C}+y_{B}^{1}+y_{D}^{1}}{3}$ & $\frac{x_{D}+y_{B}^{1}}{2}$ \\
3 & $\frac{x_{A}+3 y_{C}^{2}}{4}$ & $\frac{x_{B}+2 y_{A}^{2}+y_{D}^{1}}{4}$ & $\frac{x_{C}+3 y_{B}^{2}}{4}$ & $\frac{x_{D}+3 y_{B}^{2}-y_{D}^{1}}{6}$ \\
4 & $y_{A}^{t-1}$ & $y_{B}^{t-1}$ & $y_{C}^{t-1}$ & $\frac{x_{D}+4 y_{B}^{3}-y_{D}^{1}}{4}$ \\
$\geq 5$ & $y_{A}^{t-1}$ & $y_{B}^{t-1}$ & $y_{C}^{t-1}$ & $y_{D}^{t-1}$ \\
\hline
\end{tabular}

Note: agents' positions in column headings refer to the network structure displayed in Figure 4 .

Let us now consider the predictions for the GBR updating rule described in equations (1) and (3). Figure 6 shows how the social influence weights

\footnotetext{
${ }^{12}$ This means that, for instance, the node that has label B and upper-right position in session 1, has then label D and bottom-left position in session 2, label $\mathrm{C}$ and bottom-right position in session 3, label A and upper-left position in session 4, respectively.
} 
for each of the four network nodes change as a function of $\rho$. When $\rho=0$, agents $B$ and $D$ are the most influential. This, loosely speaking, reflects the fact that both $B$ and $D$ have an outdegree of 2 , while $A$ and $C$ have an outdegree of 1: the agents who are listened to by more other agents are the most influential. ${ }^{13}$ Also note that $A$ is relatively more influential than $C$. This reflects indirect social influence, as $A$ communicates to $B$, who is one of the two most influential subjects, while $C$ communicates to $A$. For $\rho>0$, the pattern of social influence weights also reflects agents' indegree. In particular, as $\rho$ increases, $B$ becomes progressively more influential, while the weights of the other three agents tend to zero.

Figure 6: Social influence weights as a function of $\rho$

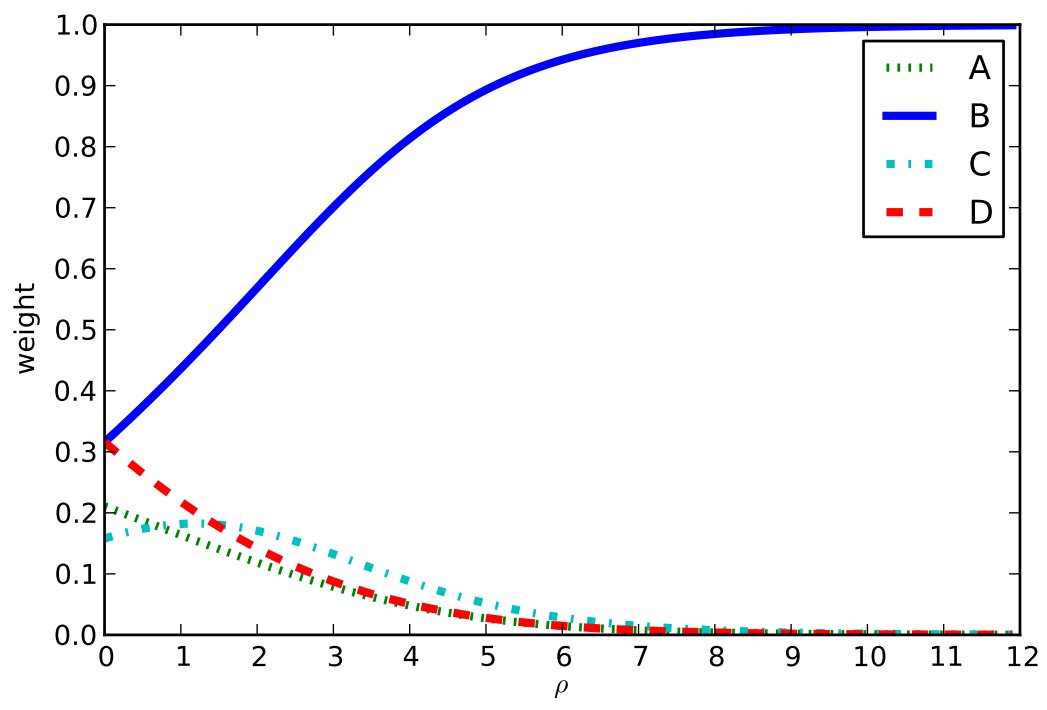

To summarize, Table 2 compares point predictions of social influence weights for $\rho=0, \rho=1$ and $\rho \rightarrow \infty$. When $\rho=0$, as in DeMarzo et al. (2003), $B$ and $D$ have equal social influence weights, similarly to the case of Bayesian updating. When $\rho=1$, so that agents update their beliefs using weights that are proportional to indegree, $B$ is the most influential agent. Finally, when $\rho \rightarrow \infty$, consensus beliefs tend to agent $B$ 's initial opinion.

The pattern described in Figure 6 and Table 2 provides the predictions to be tested in the experiment. The first hypothesis we test is that agents optimally update their beliefs. Empirically, the null hypothesis is that all

\footnotetext{
${ }^{13}$ Note that $\mathrm{B}$ and D have exactly the same weight since they communicate to the same individuals ( $C$ and each other) and, while the link from $A$ to $B$ implies that the latter is placing a lower weight $\left(\frac{1}{3}\right.$ rather than $\left.\frac{1}{2}\right)$ on her own belief, she is hence placing lower weight also on the information coming from $D$.
} 
Table 2: Predictions for social influence weights for different values of $\rho$

\begin{tabular}{lcccc}
\hline \hline & $w_{A}$ & $w_{B}$ & $w_{C}$ & $w_{D}$ \\
\hline$\rho=0$ & 0.21 & 0.32 & 0.16 & 0.32 \\
$\rho=1$ & 0.18 & 0.39 & 0.18 & 0.26 \\
$\rho \rightarrow \infty$ & 0.00 & 1.00 & 0.00 & 0.00 \\
\hline
\end{tabular}

Note: the predictions refer to the updating rule described in equations (1) and (3) for the network structure displayed in Figure 4.

nodes have equal weights in consensus beliefs:

$$
H_{0}: w_{A}=w_{B}=w_{C}=w_{D}=0.25
$$

Note that this is a general test of Bayesian updating versus an unspecified alternative. In order to test against the specific alternative of the generalized boundedly rational updating rule, we focus on pair-wise differences between individual weights. More specifically, as shown in Figure 6, the updating rule predicts that, for any $\rho \geq 0$, an agent in node $B$ is more influential than in either $A$ or $C$. Conversely, for all other pair-wise comparisons between nodes, the sign of the difference between weights is not independent of $\rho$. Therefore, the relevant one-sided hypotheses can be stated as follows:

$$
\begin{aligned}
& H_{0}: w_{B} \leq w_{A} \quad \text { vs } H_{1}: w_{B}>w_{A} \\
& H_{0}: w_{B} \leq w_{C} \text { vs } H_{1}: w_{B}>w_{C}
\end{aligned}
$$

Next, we focus on the value of $\rho$. For $\rho=0$, analogously to the case of Bayesian updating, the boundedly rational updating rule predicts $w_{B}=w_{D}$. On the other hand, for $\rho>0$, the rule predicts $w_{B}>w_{D}$ (see Figure 6). We can thus test the effect of indegree on social influence $(\rho>0)$, versus the alternative of no effect $(\rho=0)$, by comparing the social influence weights of agents $B$ and $D$ :

$$
H_{0}: w_{B} \leq w_{D} \quad \text { vs } \quad H_{1}: w_{B}>w_{D}
$$

\subsection{Procedures}

The experiment was conducted in the Experimental Economics Lab of the University of Milan Bicocca between January and March 2013, with 24 subjects participating in each of the four sessions (96 in total). Subjects were undergraduate students, recruited by e-mail through an online system. The experiment was ran using z-Tree (Fischbacher, 2007). Subjects on average 
earned 13.8 euro for sessions lasting approximately 80 minutes, including time for instructions, control questions and payments.

Each session consisted of four eight-round phases. Subjects were informed that signals and network positions would be randomly determined at the beginning of each phase, while the composition of the groups would remain the same throughout the session. Subjects were only informed that the signals would be integer numbers randomly drawn by the system. The four signals for each group/phase were extracted as follows. An integer number $\theta$ was extracted from a uniform distribution in a range between 200 and 800 . Four positive integers were then randomly drawn from a normal distribution with mean $\theta$ and variance 100 .

All the experimental instructions, reported in Appendix B, were provided to the participants in written form, and also read aloud at the beginning of the session. Individuals were then asked to answer some control questions. Each participant had the possibility to take notes and make calculations on paper, and also to use an on-screen calculator. Moreover, in each round, the screen reported all the information available (own past guesses and past guesses of neighbors since the beginning of the phase), in order to guarantee perfect recall.

The instructions explicitly suggested that, had an individual known with certainty a subset of the signals for her group, her optimal strategy was to report their average. This, together with the fact that individuals had to target the average of four specific numbers (rather than the mean of an underlying distribution of signals) helped us to minimize mistakes caused by inappropriate statistical inference, hence ensuring that individuals could focus on the process of information aggregation.

\section{Results}

In each of the four sessions, the experimental task was implemented by 24 subjects over 8 rounds in four different phases (32 rounds in total), resulting in 384 observations for each round $(24$ subjects $\times 4$ sessions $\times 4$ phases $)$ and 3072 observations in total. Overall, although there was substantial heterogeneity at individual and group level, subjects generally showed to have clearly understood the experimental task. In the first round of each phase, 94.2 per cent of the subjects truthfully reported their own signal, while 96 per cent of the subjects reported a number within 10 units from their own signal. In the final round of each phase, 24 per cent of the subjects correctly guessed the average of the four signals within their group. Accounting for rounding errors, 55.5 per cent of the subjects reported beliefs within 10 units from the average of the four signals. 


\subsection{Tests of Hypotheses}

In order to test hypotheses about the social influence weights of agents in different network positions, ${ }^{14}$ we specify each agent's final belief as a linear combination of the initial signals of the four agents in her group:

$$
y_{i}^{T}=\mu+w_{A} x_{i, A}+w_{B} x_{i, B}+w_{C} x_{i, C}+w_{D} x_{i, D}+\varepsilon_{i}
$$

where $y_{i}^{T}$ is agent $i$ 's belief in the last round of each phase, $x_{i, j}$ is the signal observed by agent $j$ in $i$ 's group, $\mu$ is a constant, $w_{j}$ is the social influence weight of agent $j$, and $\varepsilon_{i}$ is an idiosyncratic error term. Equation (10) is estimated by OLS, under the constraint $\sum_{j} w_{j}=1$. The set of regressors also includes full sets of dummy variables for sessions and phases. In order to take into account the dependence of observations belonging to the same group within each session, standard errors are clustered by 24 independent groups (there are 6 independent groups in each of the four sessions).

Table 3 presents the results. Since we are focusing on the final observation from each of the four phases, the overall sample includes 384 individual observations. Column (1) reports estimates of social influence weights in absolute terms, as in Equation (10). The weights generally differ from 0.25, with a pattern that is qualitatively consistent with the predictions of the generalized updating rule: social influence is highest for node $B(0.294)$ and lowest for node $\mathrm{C}(0.214)$. The hypothesis that all nodes have equal weights $\left(w_{A}=w_{B}=w_{C}=w_{D}=0.25\right)$, as predicted under Bayesian updating, is strongly rejected by the data $(p<0.01)$.

Result 1: Bayesian updating is rejected by the data.

Focusing on pair-wise differences between weights (hypothesis H2-H3), $w_{B}$ is higher than $w_{C}$, consistent with the predictions of the generalized boundedly rational rule, and the difference $(+0.080)$ is strongly significant $(p<0.01)$. Similarly, $w_{B}$ is higher than $w_{A}$, although the difference $(+0.026)$ is not statistically significant $(p<0.25)$.

Result 2: Pairwise differences between social influence weights are consistent with the GBR updating rule.

Next, turning to $\mathrm{H} 4$, we find that $w_{B}$ is higher than $w_{D}$ and the difference is strongly significant $(p<0.01)$. This leads us to reject the null hypothesis that $\rho=0$.

\footnotetext{
${ }^{14}$ Throughout the discussion of the results, unless otherwise stated, we will refer to the four network nodes using the labels of session 1 (see Figure 5). This means that nodes from sessions 2 to 4 are implicitly relabeled so that they are the same as in session 1 .
} 
Table 3: Estimated social influence weights, overall

\begin{tabular}{lcc}
\hline \hline & $(1)$ & $(2)$ \\
& Absolute weights & Relative weights \\
\hline Signal A & $0.268^{* * *}$ & 0.018 \\
& $(0.031)$ & $(0.031)$ \\
Signal B & $0.294^{* * *}$ & $0.044^{* * *}$ \\
& $(0.013)$ & $(0.013)$ \\
Signal C & $0.214^{* * *}$ & $-0.036^{* *}$ \\
& $(0.017)$ & $(0.017)$ \\
Signal D & $0.224^{* * *}$ & -0.026 \\
& $(0.023)$ & $(0.023)$ \\
\hline Number of observations & 384 & 384 \\
\hline Note: figures reported are OLS estimates of social influence weights associated to the \\
node indicated by the row heading. The weights are expressed in absolute terms (column \\
1) and as a difference from 0.25 (column 2), respectively. Dependent variable: individual \\
beliefs in final round. All specifications include full sets of session and phase dummies. \\
Standard errors clustered at group level reported in brackets. ${ }^{* * *} \mathrm{p}<0.01,{ }^{* *} \mathrm{p}<0.05$, \\
${ }^{*} \mathrm{p}<0.10$.
\end{tabular}

Result 3: The social influence of an individual is positively affected by the number of individuals she listens to.

This finding is important, as it indicates that subjects do not place equal weights on all their neighbors, but take into account their neighbors' indegree when aggregating the information they receive from them. As a result, ceteris paribus, subjects with higher indegree ultimately have higher social influence.

In order to shed light on these findings, column (2) reports differences of social influence weights with respect to 0.25 , obtained by expressing individual final-round beliefs as deviations from the average of the four group signals. The results indicate that $w_{B}$ is significantly higher than $0.25(p<0.01)$, while $w_{C}$ is significantly lower than $0.25(p<0.02)$. On the other hand, $w_{A}$ and $w_{D}$ are not significantly different from $0.25(p<0.29$ and $p<0.13$, respectively, for the corresponding one-sided hypothesis). The different test results for nodes $B$ and $D$ provide further evidence against a simple updating rule à la DeMarzo et al. (2003).

\subsection{Robustness}

In order to assess the robustness of the results to the possible effects of outliers, Table 4 reports estimates of (relative) social influence weights obtained by eliminating from the sample the groups containing the $1 \%, 5 \%$, or $10 \%$ most extreme observations, where potential outliers are identified by consid- 
ering, for each group member, the difference between the reported beliefs and the ones predicted by Bayesian updating. This results in a restricted sample size of 380, 364 and 344 observations, respectively. In all cases, the estimates are virtually unchanged relative to the overall sample. The hypothesis that all nodes have equal weights $\left(w_{A}=w_{B}=w_{C}=w_{D}=0.25\right)$ is strongly rejected by the data $(p<0.01)$. The hypothesis that $w_{B}=w_{D}$ is also strongly rejected in all cases. Indeed, by eliminating potential outliers, the estimated weights are even more closely consistent with the theoretical predictions of the generalized boundedly rational updating rule. In column (3), for example, where the 10 per cent of the groups reporting the most extreme deviations from optimal predictions are excluded, the estimated relative weights are $0.005,0.053,-0.045$ and -0.012 . In all cases, $w_{B}\left(w_{C}\right)$ is significantly higher (lower) than 0.25 .

Table 4: Social influence (relative weights), robustness

\begin{tabular}{lccc}
\hline \hline & $(1)$ & $(2)$ & $(3)$ \\
& $1 \%$ & $5 \%$ & $10 \%$ \\
\hline Signal A & 0.013 & 0.019 & 0.005 \\
Signal B & $(0.031)$ & $(0.032)$ & $(0.027)$ \\
& $0.049^{* * *}$ & $0.051^{* * *}$ & $0.053^{* * *}$ \\
Signal C & $(0.014)$ & $(0.015)$ & $(0.014)$ \\
& $-0.037^{* *}$ & $-0.045^{* *}$ & $-0.045^{* * *}$ \\
Signal D & $(0.017)$ & $(0.018)$ & $(0.017)$ \\
& -0.026 & -0.024 & -0.012 \\
& $(0.023)$ & $(0.023)$ & $(0.017)$ \\
\hline Number of observations & 380 & 364 & 344
\end{tabular}

Note: the figures reported are estimates of social influence weights, as a difference from 0.25 , associated to the subject in the position indicated by the row heading. Dependent variable: individual beliefs in final round. All specifications include full sets of session and phase dummies. Standard errors clustered at group level reported in brackets.

${ }^{* * *} \mathrm{p}<0.01,{ }^{* *} \mathrm{p}<0.05,{ }^{*} \mathrm{p}<0.10$. Columns (1) to (3): sample restricted by eliminating groups with most extreme deviations from optimal beliefs $(1 \%, 5 \%, 10 \%$, respectively).

It should be observed that, although the variance of beliefs held by the four group members falls steadily over successive rounds in all groups, disagreement persists in many cases, so that beliefs do not converge to a consensus in all cases. In order to assess the potential effects of non-convergence, Table 5 presents estimates of (relative) social influence weights by individual network position. Focusing on nodes $B$ and $D$, in columns (2) and (4), respectively, estimated social influence weights are qualitatively unchanged with respect to the overall results in Table 3: the relative weight of $B$ is positive and significant, while it is negative and significant for $C$. Agent $D$ 
has a negative relative weight in the final beliefs of agent $A$. Finally, relative social influence weights are not different from zero for $C$.

Table 5: Social influence (relative weights), by node

\begin{tabular}{lcccc}
\hline \hline & $(1)$ & $(2)$ & $(3)$ & $(4)$ \\
& Node A & Node B & Node C & Node D \\
\hline Signal A & 0.057 & 0.015 & 0.007 & -0.009 \\
& $(0.047)$ & $(0.037)$ & $(0.040)$ & $(0.066)$ \\
Signal B & 0.038 & $0.057^{* *}$ & 0.015 & $0.065^{* *}$ \\
& $(0.038)$ & $(0.024)$ & $(0.023)$ & $(0.026)$ \\
Signal C & 0.013 & $-0.070^{* *}$ & 0.024 & $-0.110^{* * *}$ \\
& $(0.033)$ & $(0.027)$ & $(0.022)$ & $(0.035)$ \\
Signal D & $-0.109^{* *}$ & -0.003 & -0.045 & 0.053 \\
& $(0.041)$ & $(0.025)$ & $(0.031)$ & $(0.048)$ \\
\hline Number of observations & 96 & 96 & 96 & 96 \\
\hline
\end{tabular}

Note: the figures reported are estimates of the social influence weights, as a difference from 0.25 , associated to the subject in the position indicated by the row heading. Dependent variable: individual beliefs in final round. All specifications include full sets of session and phase dummies. Standard errors clustered at group level reported in brackets. ${ }^{* * *} \mathrm{p}<0.01,{ }^{* *} \mathrm{p}<0.05,{ }^{*} \mathrm{p}<0.10$.

Overall, these results indicate that the effects of network structure on social influence reported in Section 4.1 are both qualitatively and quantitatively robust to the potential effects of outliers. In addition, they are qualitatively unaffected by the possible non-convergence of beliefs within individual groups.

\subsection{Further Evidence}

The experimental data also allow us to investigate what explains the treatment effects on social influence weights, by looking at how agents at specific nodes aggregate the information they receive in each round. At individual level, there can be two possible, not mutually exclusive, mechanisms explaining differences in social influence between $B$ and $D$. The first, and most intuitive, mechanism is that may $C$ place a higher weight on the opinion of $B$ than on the one of $D$, because of $B$ 's higher indegree. The second is that, for the same reason, $D$ may be influenced by $B$ relatively more than $B$ is influenced by $D$.

Table 6 sheds light on this issue by presenting estimates of node-specific (absolute) weights based on all updating rounds. Looking at the estimates for node $C$ (column 3 ), the weight of $B(0.435)$ is substantially higher than the one of $D(0.163)$, and the difference is strongly statistically significant $(p<$ 
0.01). This provides support to the first of the two mechanisms described above. The comparison of the weights given to each other by $B$ and $D$ is non-trivial, since their respective indegrees are different, as they form their beliefs on the basis of different numbers of neighbors. However, $D$ appears to substantially under-weigh the information coming from $B(0.104)$, whereas $B$ does not substantially under-weigh the information received from $D(0.325)$. The overall effect of indegree on social influence is therefore mainly explained by the way in which information is processed by $C$ : node $B$, whose indegree is twice the indegree of $D$, receives a weight that is more than twice as large as the weight for node $D$.

Table 6: Neighbors' absolute weights in current beliefs, by node

\begin{tabular}{|c|c|c|c|c|}
\hline & $\begin{array}{c}(1) \\
\text { Node A }\end{array}$ & $\begin{array}{c}(2) \\
\text { Node B }\end{array}$ & $\begin{array}{c}(3) \\
\text { Node C }\end{array}$ & $\begin{array}{c}(4) \\
\text { Node D }\end{array}$ \\
\hline Lagged belief, node A & $\begin{array}{c}0.530^{* *} \\
(0.011)\end{array}$ & $\begin{array}{c}0.000 \\
(0.000)\end{array}$ & & \\
\hline Lagged belief, node B & & $\begin{array}{c}0.675^{* *} \\
(0.070)\end{array}$ & $\begin{array}{c}0.435^{* *} \\
(0.087)\end{array}$ & $\begin{array}{c}0.104^{* *} \\
(0.018)\end{array}$ \\
\hline Lagged belief, node C & $\begin{array}{c}0.470 * * \\
(0.011)\end{array}$ & & $\begin{array}{c}0.402^{* *} \\
(0.087)\end{array}$ & \\
\hline Lagged belief, node D & & $\begin{array}{c}0.325^{* *} \\
(0.070)\end{array}$ & $\begin{array}{c}0.163^{* *} \\
(0.049)\end{array}$ & $\begin{array}{c}0.896^{* *} \\
(0.018)\end{array}$ \\
\hline Number of observations & 672 & 672 & 672 & 672 \\
\hline
\end{tabular}

Finally, since the hypothesis that $\rho=0$ is strongly rejected, it is interesting to ask what value of $\rho$ provides the best fit for the experimental data. We simulated the generalized updating rule with a wide range of values for $\rho$, searching for the value that minimizes the sum of squared deviations, over all individuals, between observed (experimental) and simulated final-round beliefs:

$$
\hat{\rho}=\underset{\rho}{\arg \min } \sum_{g=1}^{96} \sum_{k=1}^{4}\left(y_{g, k, T}-\bar{y}_{k, T}^{\rho}\right)^{2},
$$

where $y_{g, k, T}$ is the belief of an individual with role $k$ in group $g$ in the final round, and $\bar{y}_{k, T}^{\rho}$ is the corresponding theoretical prediction. This produces an estimate of $\hat{\rho}=0.30$. Interestingly, this is higher than $\rho^{*}=0.04$, the value of $\rho$ that provides the best approximation to the results of the optimal 
strategy (see Figure 3). This indicates that agents, as they should, place higher weight on those neighbors who themselves listen to more peers, but they do so to a greater extent than would be optimal.

\section{Conclusions}

Although the mathematical concept of digraph, i.e., a network based on $d i$ rected relations, was already central in the pioneering works of French (1956) and Harary (1959), empirical studies of information diffusion in social networks have generally not focused explicitly on the respective roles played by indegree and outdegree. This may be partly reflecting the fact that, although asymmetric information flows are the norm in opinion formation, most of the available network data sets (such as those describing friendship relations on online social networks, co-authorships of academic authors, or traffic flows) generally describe undirected networks. Recently, however, increasing attention has been given, both theoretically and empirically, to information flows in directed networks (e.g. Baños et al., 2013 and Gleeson et al., 2013).

This paper investigated a boundedly rational model of opinion formation in directed social networks that provides a simple generalization of the linear updating rules in DeGroot (1974) and DeMarzo et al. (2003). In the model, agents aggregate the information they receive from their neighbors' by using weights that may reflect their neighbors' indegree. Intuitively, when opinions are updated, relatively more importance can be attributed to more informed individuals.

At the theoretical level, our results indicate that, in balanced networks, placing higher weight on neighbors with higher indegree is generally less efficient than placing equal weights on all neighbors. On the other hand, in unbalanced networks, it can be efficient to place higher weight on neighbors with higher indegree. Indeed, there exist unbalanced networks in which the optimal importance attributed to indegree is arbitrarily high. At the empirical level, our experimental results provide clean evidence of a causal effect of indegree on social influence. Both Bayesian updating and boundedly rational updating à la DeMarzo et al. (2003) are rejected against the alternative of a boundedly rational updating rule in which the weight placed on an agent's opinion is positively related to the number of individuals she listens to. Indeed, the importance that agents place on their neighbors' indegree is higher than would be efficient.

One possible interpretation of our findings is that agents are aware that, in the setting considered, placing a higher weight on neighbors with a higher indegree is efficient. However, in their attempt to aggregate information efficiently while retaining a simple updating rule, agents end up placing excessive weight on neighbors with high indegree. A second possible explanation 
is that, irrespective of any efficiency motivation, agents tend to attribute some form of authority to peers whom they perceive as better informed, and this leads them to place a higher weight on the information received from them. Another possible interpretation of our results is that the weights of the updating rule could be state-dependent. In the framework by Hegselmann and Krause (2002), for example, updating weights depend negatively on the distance between opinions. Since the beliefs of high-indegree agents are, on average, less extreme than those of low-indegree agents, they can be expected to be more similar, on average, to the beliefs of the listening agents. In this perspective, our results could be interpreted as reflecting features of beliefs, so that network structure, and more specifically indegree, would play a role only indirectly.

To sum up, our analysis provides causal evidence of an indegree effect that is at odds with the updating mechanisms most commonly adopted in the literature on opinion dynamics. When forming their opinion, agents do not place equal weights on all their neighbors, but use weights that are positively related to their neighbors' indegree. As a result, ceteris paribus, subjects with higher indegree ultimately have higher social influence. This is an important finding, as it indicates that, despite their inability to fully account for the structure of their communication network, agents are able to exploit the information about its local properties. Further research should contribute to an understanding of the mechanisms explaining the effect of indegree on opinion formation and social influence. 


\section{Appendix A - Proofs}

Proof of Theorem 1. Consider a strongly connected network $\mathcal{G}$ : its adjacency matrix $M_{\mathcal{G}}=q_{i j_{i, j \leq N}}$ is necessarily irreducible. ${ }^{15}$ Perfect and Mirsky (1965) have shown that then there exists another matrix $P_{\mathcal{G}}$, with coefficients $p_{i j}$ such that

- $P_{\mathcal{G}}$ is doubly stochastic. ${ }^{16}$

- $p_{i j}=0 \Longleftrightarrow q_{i j}=0$.

The coefficients $p_{i j}$ define a new linear updating rule applicable to the network $\mathcal{G} .{ }^{17}$ Let us calculate the dynamics of the average of beliefs from one period to another according to this new updating rule:

$$
\begin{aligned}
\frac{1}{N} \sum_{i} y_{i}^{t+1} & =\frac{1}{N} \sum_{i} \sum_{j} p_{i j} y_{j}^{t} \\
& =\frac{1}{N} \sum_{j} \underbrace{\sum_{i} p_{i j}}_{=1} y_{j}^{t} \\
& =\frac{1}{N} \sum_{j} y_{j}^{t} .
\end{aligned}
$$

Such average is unchanged. By iterating this reasoning, we have that the average of opinions at any time is equal to the initial mean $\bar{x}$. When a consensus is reached, it is by definition the correct consensus.

Proof of Theorem 2. Given a network $\mathcal{G}$, let $\overline{\mathcal{G}}$ be the corresponding undirected network (with adjacency matrix $\bar{q}_{i j}=1 \Longleftrightarrow q_{i j}+q_{j i}>0$ ), and $\mathcal{G}_{i}{ }^{\delta}$ the subnetwork of $\mathcal{G}$ restricted to nodes which are distant at most $\delta \in \mathbb{N}$ from $i$ in $\overline{\mathcal{G}}$. Since the weights $\bar{\pi}_{i j}$ only depend on local properties of the network, there must exist a $\bar{\delta} \in \mathbb{N}$ such that they are only determined based on $\mathcal{G}_{i}^{\bar{\delta}}$. Consider then the networks in figure 7 :

Notice that $\mathcal{G}_{E}^{\bar{\delta}}$ is identical in both networks, and hence the vector of weights $\bar{\pi}_{E}$ adopted by $E$ must be identical too. Assume, without loss of generality, that $\bar{\pi}_{E A_{\bar{\delta}}} \geq \bar{\pi}_{E C_{\bar{\delta}}}$. In the second network, $E$ gets to know the

\footnotetext{
${ }^{15} \mathrm{An} N \times N$ matrix $q$ is reducible if the set $\{1, \ldots, N\}$ can be partitioned in two subsets $V_{1}, V_{2}$ such that $q_{i j}=0$ whenever $i \in V_{1}$ and $j \in V_{2}$. This implies that nodes in $V_{1}$ are not connected to nodes in $V_{2}$, and hence that the network is not strongly connected.

${ }^{16} \mathrm{~A}$ matrix is doubly stochastic if all its elements are non negative, and each row and column sums up to 1 .

${ }^{17}$ The relation between the double stochasticity of the updating matrix and the correctness of the consensus was already recognized by Harary (1959). The rest of the proof simply generalizes his Theorem 14 to a generic linear rule.
} 
Figure 7: Locally similar networks for node $E$

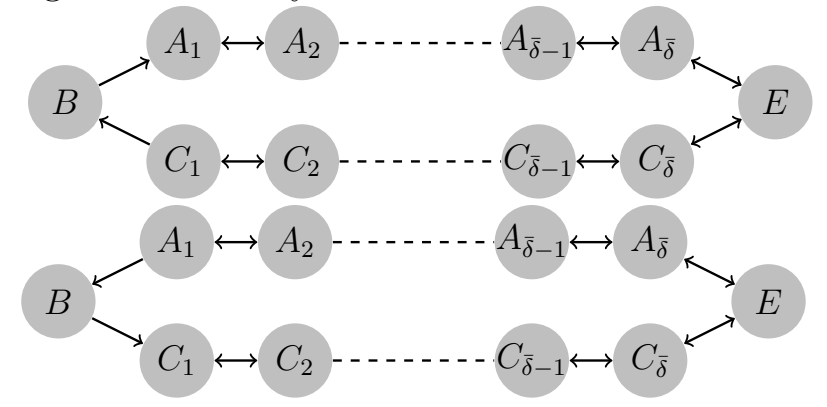

opinion of agents $B, C_{1}, \ldots, C_{\delta}$ only through the link coming from $C_{\delta}$. Hence, she is weighting the opinions of those $\bar{\delta}+1$ nodes less than the opinions of the other $\bar{\delta}$ nodes $A_{1}, \ldots, A_{\bar{\delta}}$. Hence, this rule cannot lead to the correct consensus on such network.

Example 1 Consider a network $\mathcal{G}$ with agents numbered from 1 to $N$, and execute the following steps:

1. start from agent 1: since the network is strongly connected, there must be a path from 1 to 2: call it $s_{1}$, and assume without loss of generality that it has no cycles;

2. again, since the network is strongly connected, there must be a path from 2 to 3: assume without loss of generality that it has no cycles, and call $s_{2}$ the union of $s_{1}$ with such a path;

3. by repeating the step above, for each $i<N$, a path $s_{i}$ is constructed, which goes from 1 to $i$ and passes through any $i^{\prime}<i$ : let $s_{N}$ be the union of $s_{N-1}$ with a path (again, without cycles) from $N$ to $1: s_{N}$ is a cycle which passes through each node at least once and at most $N$ times;

4. for each pair $(i, j)$ with $j \neq i$, define $\pi_{i j}$ as $\frac{1}{N}$ multiplied by the number of times that $s_{N}$ passes through the link from $j$ to $i$ (possibly zero). For each $i$, define $\pi_{i i}$ as $1-\sum_{j \neq i} \pi_{j i}$.

The updating rule having such $\pi_{i j}$ as updating weights is a valid linear rule. If we consider $s_{N}$ as a weighted network where the weight of a link is given by $\pi_{i j}$, it is strongly connected, and its adjacency matrix is doubly stochastic. Hence, the resulting updating rule leads to the correct consensus.

\section{Proof of Lemma 1.}


Consider the influence weights for the opinion of a given agent as a vector in the standard $n-1$-simplex, $\omega_{i j}^{t}$. For instance,

$$
\omega_{i}^{0}=(\underbrace{0, \ldots, 0}_{i-1}, 1, \underbrace{0, \ldots, 0}_{N-i})
$$

that is, before receiving any information from neighbors, the opinion of each agent is entirely formed by her initial signal. With a linear belief updating rule, the evolution of such vectors is simply described as a straightforward rewriting of $(1)$ :

$$
\begin{aligned}
\omega_{i}^{t} & =\sum_{j=1}^{n} q_{i j} \pi_{i j} \omega_{j}^{t-1} \\
& =\pi_{i i} \omega_{i}^{t-1}+\sum_{j \neq i} q_{i j} \pi_{i j} \omega_{j}^{t-1} .
\end{aligned}
$$

Since the network is anonymous, all neighbors of a given node are equivalent; and since the rule is anonymous, each node hence places an equal weight to each neighbor. So the above can be rewritten as

$$
\omega_{i}^{t}=\pi_{i i} \omega_{i}^{t-1}+\frac{\left(1-\pi_{i i}\right)}{d_{i}} \sum_{j \neq i} q_{i j} \omega_{j}^{t-1},
$$

where $d_{i}$ is agent $i$ 's indegree. Notice that $y_{i}^{t}=\omega_{i}^{t} \cdot x$, and hence the beliefs are uniquely determined once $\pi_{i i}^{t}$ is fixed for each $i$.

For what concerns point 2, let us define the system as biased at a given time $\bar{t}$ if for some $h$

$$
\sum_{i=1}^{N} \omega_{i h}^{\bar{t}} \neq 1
$$

Let us assume without loss of generality that $\bar{t}$ is the first time for which this happens. Notice that $\bar{t}>0$, since

$$
\sum_{i=1}^{N} \omega_{i h}^{0}=\omega_{h h}^{0}=1,
$$

and that

$$
\begin{aligned}
\sum_{i=1}^{N} \omega_{i h}^{\bar{t}} & =\sum_{i=1}^{N} \sum_{j=1}^{N} q_{i j} \pi_{i j} \omega_{j h}^{\bar{t}-1} \\
& =\sum_{j=1}^{N} \omega_{j h}^{\bar{t}-1} \sum_{i=1}^{N} q_{i j} \pi_{i j} .
\end{aligned}
$$


Since the network and the rule are anonymous, the value of the nested sum must be the same for all $j$, and since the sum of $q_{i j} \pi_{i j}$ across $j$ and $i$ is $N$, the value of such nested sum must be $\frac{N}{N}=1$. So

$$
\sum_{j=1}^{N} \omega_{j h}^{\bar{t}-1}=\sum_{i=1}^{N} \omega_{i h}^{\bar{t}} \neq 1 .
$$

But this contradicts the hypothesis that $\bar{t}$ is the first time at which the system is biased.

Proof of Theorem 4. One implication is obvious: given any agent $i$, let $\bar{d}$ be the (equal, by assumption) indegree of all $d_{i}+1$ agents in $S(i)$. Then, by applying Equation (3), we have that for each $j \in S(i)$,

$$
\pi_{i j}=\frac{\bar{d}^{\rho}}{\left(d_{i}+1\right) \bar{d}^{\rho}}=\frac{1}{d_{i}+1},
$$

that is, the updating weights do not depend on the value of $\rho$ - hence the consensus belief and social influence weights do not either. For the reverse implication, notice that if a network $\mathcal{G}$ is not regular, there is at least a pair of agents $i, j$ with different indegree and such that $i$ listens to $j$ (if this is not the case, it is easily to show by induction that the network is regular). Define now as $H_{k}$ the set of agents $h$ such that there exist $j^{\prime}, j^{\prime \prime} \in S(h)$ with $k=d_{j^{\prime}}<d_{j^{\prime \prime}}$. Intuitively, we are considering all agents $i$ on whose updating weights $\rho$ does matter, because the neighbors have different indegree, and classifying them based on the lowest indegree of a neighbor: the underlying idea of the remaining of the proof is that this will allow us to identify an agent who is necessarily disadvantaged, in terms of social influence, by a strictly positive value of $\rho$. Let $\bar{k}$ be the smallest $k$ such that $H_{k}$ is non-empty the non-regularity assumption means precisely that there exists at least one such $k$. Let $\bar{i} \in H_{\bar{k}}$, and $\bar{j} \in S(i)$ such that $d_{\bar{j}}=\bar{k}$. Notice that, for each $i^{\prime} \in S^{-1}(\bar{j})$, we have that $\bar{j}$ must have smaller or equal indegree than all other agents in $S\left(i^{\prime}\right)$ (otherwise, we would have found a non-empty $H_{k^{\prime}}$ with $\left.k^{\prime}<\bar{k}\right)$. As a consequence, $\pi_{i^{\prime} j}$ will be weakly smaller with $\rho>0$ than with $\rho=0$, and $\pi_{i \bar{j}}$ will be strictly smaller. But since we know that

$$
w_{\bar{j}}=\sum_{i=1}^{N} \pi_{i \bar{j}} w_{i},
$$

this means that $w_{\bar{j}}$ will strictly decrease as a function of its neighbors, and hence that at least some $w_{i}$ will be affected by a change of $\rho$.

Proof of Lemma 5. Let $\mathcal{G}$ be a balanced network with adjacency matrix $q$. Under $\rho=0$ the social influence of an agent is positively related to her 
outdegree and the outdegree of the agents she talks to - the entity of the relation being given precisely by the product of the updating coefficients along each possible path between the two agents. Instead, the effect of an increase in $\rho$ on the social influence of an agent $i$ is clearly related to her indegree. However, in a balanced network, the indegree and outdegree coincide. This implies that an increase in $\rho$ will amplify the vector of absolute differences between $w$ and the vector of unbiased weights $\left(\frac{1}{N}, \ldots, \frac{1}{N}\right)$ and hence decrease efficiency (defined as the sum of squares of such absolute differences).

\section{Proof of Theorem 6.}

Given a natural number $K$, consider a network having the following binary tree-like structure:

- an agent $A_{1}$ listens to two other agents $A_{2,1}, A_{2,2}$,

- each agent $A_{k, i}$ listens to two other agents $A_{k+1,2 i-1}, A_{k+1,2 i}$, for each $k<K$,

- each "leaf" agent $A_{K, i}$ listens to $A_{1}$ and to her "close relatives" $A_{K, i-1}$ and $A_{K, i+1}\left(A_{K, 1}\right.$ listens to $A_{K, 2^{K}}$ and $A_{K, 2}$, while $A_{K, 2^{K}}$, listens to $A_{K, 2^{K}-1}$ and $\left.A_{K, 1}\right)$.

Notice that,

- the structure is perfectly symmetric, in the sense that all the agents positioned on a given "layer" will exhibit the same vector of updating weights (which we will hence denote for simplicity as $\pi_{k-1, k}$ and $\pi_{k, k}$ rather than $\pi_{A_{k-1, i} A_{k, j}}$ and $\pi_{A_{k, i} A_{k, i}}$, respectively) and the same social influence (which we will hence denote as $w_{k}$ rather than $w_{A_{k, i}}$ ),

- for most of the layers of this structure, the updating weights are independent from $\rho$; namely, for any $k$ such that $1 \leq k<K-1$,

$$
\pi_{k, k}=\pi_{k-1, k}=\frac{2^{\rho}}{3 \cdot 2^{\rho}}=\frac{1}{3}
$$

and hence

$$
\begin{aligned}
w_{k} & =\pi_{k, k} w_{k}+\pi_{k-1, k} w_{k-1} \\
& =\frac{1}{3} w_{k}+\frac{1}{3} w_{k-1} \\
& =\frac{1}{2} w_{k-1}
\end{aligned}
$$

- as a consequence, since the number of agents on a given layer double at each level, this means that the sum of social influences of all agents in a given layer, which we will denote as $W_{k}$, is the same for any $k$ from 1 to $K-1$. 
Now consider the social weight of $A_{1}$. The updating weights of a leaf (which has indegree 3 , rather than 2 ) can be calculated as:

$$
\pi_{K, 1}=\frac{2^{\rho}}{2^{\rho}+3^{\rho}+3^{\rho}+3^{\rho}}=\frac{2^{\rho}}{2^{\rho}+3^{\rho+1}} ; \quad \pi_{K, K}=\frac{3^{\rho}}{2^{\rho}+3^{\rho+1}} .
$$

and since $2^{K}$ leafs listen to $A_{1}$,

$$
\begin{aligned}
w_{1} & =\pi_{1,1} w_{1}+2^{K} \pi_{K, 1} w_{K} \\
& =\frac{1}{3} w_{1}+2^{K} \frac{2^{\rho}}{2^{\rho}+3^{\rho+1}} w_{K} \\
& =2^{K} \frac{3 \cdot 2^{\rho-1}}{2^{\rho}+3^{\rho+1}} w_{K} \\
\Longrightarrow W_{1} & =\frac{3 \cdot 2^{\rho-1}}{2^{\rho}+3^{\rho+1}} W_{K} .
\end{aligned}
$$

Assume the optimal level of $\rho$ is bounded above by some $\hat{\rho}$. This means that for $K \rightarrow \infty$, this last ratio will tend to a finite limit. That is,

$$
W_{K} \sim W_{1}=W_{2}=\cdots=W_{K-1}
$$

and hence, since the sum of all $w_{i}$ is $1, w_{1}=W_{1}$ will converge to 0 asymptotically as $\frac{\alpha}{K}$, where $\alpha$ is a constant. Now, it easy instead to verify that since, for given $\bar{K}$,

$$
2^{\bar{K}} \frac{3 \cdot 2^{\rho-1}}{2^{\rho}+3^{\rho+1}} \stackrel{\rho \rightarrow \infty}{\rightarrow} 0
$$

and such term is continuous in $\rho$, we can define $\rho_{\bar{K}}$ such that

$$
2^{\bar{K}} \frac{3 \cdot 2^{\rho_{\bar{K}}-1}}{2^{\rho_{\bar{K}}}+3^{\rho_{\bar{K}+1}}}=1 ;
$$

it is easy to see that $\rho_{\bar{K}} \stackrel{\bar{K} \rightarrow \infty}{\rightarrow} \infty$. When the GBR rule is applied with such $\rho_{K}$, we have, by definition, that $w_{1}=w_{K}$. That is,

$$
\frac{W_{K}}{2^{K}}=W_{1}=W_{1}=W_{2}=\cdots=W_{K-1} .
$$

The sum of the weights still sums up to 1 , but now the nodes with the maximum influence are $2^{K}+1$ (all leafs, and $A_{1}$ ) so now each influence weight will converge to 0 as $\frac{1}{2^{K}}$ (or faster), rather than as $\frac{\alpha}{K}$.

Now, observe that the correct weights converge to 0 as $\frac{1}{N}=\frac{1}{2^{K+1}-1}$. Hence, the sum of square deviations in the case of any finite $\rho$ will converge towards at least $\left(\frac{\alpha}{K}\right)^{2}=\frac{\alpha^{2}}{K^{2}}$, while in the case of $\rho=\rho_{K}$ it will converge towards at most

$$
2^{K+1} \cdot\left(\frac{1}{2^{K}}\right)^{2}=\frac{2^{K+1}}{2^{2 K}}=\frac{1}{2^{K-1}} \stackrel{K \rightarrow \infty}{<} \frac{\alpha^{2}}{K^{2}} .
$$

Hence the most efficient $\rho$ for $K \rightarrow \infty$ must also tend to $\infty$. 
Theorem 7 Consider two strongly connected networks $\mathcal{G}_{1}, \mathcal{G}_{2}$, with adjacency matrices $q^{1}, q^{2}$, identical except for an element:

$$
0=q_{\bar{i}, \bar{j}}^{1} \neq q_{\bar{i}, \bar{j}}^{2}=1 .
$$

Let $w^{1}$ and $w^{2}$ be the vectors of social influence weights resulting from the GBR updating rule implemented with $\rho=0$ on $\mathcal{G}_{1}$ and $\mathcal{G}_{2}$, respectively. Then, $w_{\bar{j}}^{2}>w_{\bar{j}}^{\frac{1}{1}}$.

Let us define $\Delta_{i}=\frac{w_{i}^{2}-w_{i}^{1}}{w_{i}^{1}}$, the (relative) increase in the social influence of an agent $i$ when adding the link from $\bar{j}$ to $\bar{i}$ : Theorem 7 simply states that $\Delta_{\bar{j}}$ is positive. In order to prove it, we prove the following stronger result.

Lemma 2 Consider $\mathcal{G}_{1}, \mathcal{G}_{2}, w^{1}, w^{2}$, as above. Then, $\Delta_{\bar{j}} \geq \Delta_{i}$ for all $i \neq \bar{j}$.

\section{Proof of Lemma 2.}

Let $\pi_{i j}^{1}$ and $\pi_{i j}^{2}$ be the weights attributed by $i$ to $j$ in $\mathcal{G}_{1}$ and $\mathcal{G}_{2}$, respectively, and let $S_{1}(i), S_{2}(i)$ be the listening sets of $i$ in the two networks. Notice that, whenever $i \neq \bar{i}$, then $\pi_{i j}^{1}$ and $\pi_{i j}^{2}$ coincide. Equation (7) allows us to express the social influence of an agent as a linear combination of the social influence weights of the agents she talks to. By plugging it in the definition of $\Delta_{i}$, the same can be done for what concerns the relative change of influence; for any $i \neq \bar{j}$, the listeners set

$$
S^{-1}(i)=\{j: i \in S(j)\}
$$

is unchanged, and so we have

$$
\Delta_{i}=\frac{w_{i}^{2}-w_{i}^{1}}{w_{i}^{1}}=\sum_{j \in S_{1}^{-1}(i)} \frac{\pi_{j i}^{2} w_{j}^{2}-\pi_{j i}^{1} w_{j}^{1}}{w_{i}^{1}} .
$$

Let us define $\hat{\Delta}_{i}$ as:

$$
\hat{\Delta}_{i}=\sum_{j \in S_{1}^{-1}(i)} \frac{\pi_{j i}^{1}\left(w_{j}^{2}-w_{j}^{1}\right)}{w_{i}^{1}}=\sum_{j \in S^{-1}(i)} \frac{\pi_{j i}^{1} w_{j}^{1} \Delta_{j}}{w_{i}^{1}} .
$$

We can observe that:

- $\Delta_{\bar{j}}>\hat{\Delta}_{\bar{j}}$, since $S_{2}^{-1}(\bar{j})=S_{1}^{-1}(\bar{j}) \cup\{\bar{i}\}$, while $\pi_{i \bar{j}}^{1}=\pi_{i \bar{j}}^{2}$ for all $i \neq \bar{i}$,

- $\Delta_{j}<\hat{\Delta}_{j}$ for any other $j \in S_{1}^{-1}(\bar{i})$, since $\pi_{\bar{i} j}^{1}<\pi_{i j}^{2}$, while $\pi_{i j}^{1}=\pi_{i j}^{2}$ for all $i \neq \bar{i}$,

- $\Delta_{j}=\hat{\Delta}_{j}$ for any $j \notin S_{2}^{-1}(\bar{i})$. 
Now, assume Lemma 2 is false. That is, there exists $j^{\prime} \neq \bar{j}$ such that $\Delta_{j^{\prime}}>\Delta_{\bar{j}}$. Let us assume, without loss of generality, that $\Delta_{j^{\prime}} \geq \Delta_{i}$ for all $i$. We can then write:

$$
\begin{aligned}
\Delta_{j^{\prime}} \leq \hat{\Delta}_{j^{\prime}} & =\sum_{i \in S^{-1}\left(j^{\prime}\right)} \frac{\pi_{i j^{\prime}} w_{i}^{1} \Delta_{i}}{w_{j^{\prime}}^{1}} \\
& \leq \sum_{i \in S^{-1}\left(j^{\prime}\right)} \frac{\pi_{i j^{\prime}} w_{i}^{1} \Delta_{j^{\prime}}}{w_{j^{\prime}}^{1}} \\
& =\frac{\Delta_{j^{\prime}}}{w_{j^{\prime}}^{1}} \sum_{i \in S^{-1}\left(j^{\prime}\right)} \pi_{i j^{\prime}} w_{i}^{1} \\
& =\Delta_{j^{\prime}} \frac{w_{j^{\prime}}^{1}}{w_{j^{\prime}}^{1}}=\Delta_{j^{\prime}} .
\end{aligned}
$$

We can see that both inequalities must be binding. The first implies that $j^{\prime} \notin$ $S_{2}^{-1}(\bar{i})$, and hence $j^{\prime} \notin S_{1}^{-1}(\bar{i})$; the second that $\Delta_{i}=\Delta_{j^{\prime}}$ for all $i \in S^{-1}\left(j^{\prime}\right)$. By applying the same process recursively to any such $i$, and exploiting the strong connectedness of the network, we can show that $\Delta_{\bar{j}}=\Delta_{j^{\prime}}$, which contradicts the initial assumption. ${ }^{18}$

Proof of Theorem 7. The sum of social influence weights is by definition 1 in any network:

$$
\sum_{i=1}^{N} w_{i}^{1}=1=\sum_{i=1}^{N} w_{i}^{2} .
$$

As a consequence, the weighted sum of percentage changes $\Delta_{i}$ must be 0 :

$$
\sum_{i=1}^{N} w_{i}^{1} \Delta_{i}=0
$$

(with all weights $w_{i}^{1}$ strictly positive). Since $\hat{\Delta}_{i}$ is a linear combination of $\Delta_{j}$ for different $j$, if we had $\Delta_{j}=0$ for all $j$, then we would have $\hat{\Delta}_{i}=0$ for all $i$. Instead we know that $\Delta_{j}<\hat{\Delta}_{j}$ for some $j$. So the maximum $\Delta_{i}$, which is guaranteed by Lemma 2 to be $\Delta_{\bar{j}}$, must be strictly positive.

Notice that $\Delta_{\bar{i}}$ is not guaranteed to be negative. The influence of $\bar{i}$ will decrease in relative terms (that is, compared to $\hat{\Delta}_{\bar{i}}$ ), but the increase in influence of $\bar{j}$ may more than compensate this effect if there is a short path from $\bar{i}$ back to $\bar{j}$.

Similarly, $\Delta_{\bar{j}}$ is not guaranteed to be positive if $\rho>0$. Again, social influence will increase in relative terms, but for sufficiently high values of

\footnotetext{
${ }^{18}$ If the chosen path from $j^{\prime}$ to $\bar{j}$ passes through $S_{1}^{-1}(\bar{i})$, the contradiction will arise even before reaching $\bar{j}$.
} 
$\rho$ the increase in influence of $\bar{i}$ will be large enough to make the influence weights of all other agents decrease. 


\section{Appendix B - Experimental Instructions}

[Translated from Italian]

Welcome and thank you for taking part in this experiment. During the experiment talking or communicating with other participants is not allowed in any way. If you have a question at any time, raise your hand and one of the assistants will come to answer your question. By carefully following the instructions you can earn a sum of money that will depend on the choices made by you and the other participants. On top of that amount, you will receive in any case $5 €$ for the participation in this experiment.

\section{General Rules}

- 24 subjects will take part in this experiment.

- The experiment takes place in 4 phases of 8 rounds each, for a total of 32 rounds.

- At the beginning of the experiment 6 groups of four subjects will be randomly and anonymously formed by the computer.

- You will be assigned to one of the 6 groups. You will interact only with those in your group, without knowing their identity. The composition of each group will remain unchanged throughout the experiment.

\section{The development of a phase}

- In the first round of each of the four phases, in all groups, each subject will be randomly and anonymously assigned a different role: A, B, C, and D.

- The computer will randomly generate four integers that we will define as signals. Each component of the group will be shown only one of the four signals. Signals will be denoted as $x_{A}, x_{B}, x_{C}$, and $x_{D}$.

- In each of the 8 periods of the phase, each subject will be asked to guess the mean of the four signals extracted by the computer for that phase: $\bar{x}=\frac{\left(x_{A}+x_{B}+x_{C}+x_{D}\right)}{4}$.

- For making each guess, there is a maximum time of 120 seconds (which will be shown by a counter in the top right corner of the screen).

- At any moment, it is possible to open a calculator by simply clicking its icon, in the bottom left corner of the screen.

\section{How earnings are determined}


- Individual earnings will depend on how close the guess comes to the value of $\bar{x}$ :

- At the end of the experiment, the computer will randomly extract one of the 32 periods.

- The earnings will be equal to 15 euros minus the difference (in absolute value) between $\bar{x}$ and the guess made in the selected round.

- If this difference turns out to be negative, the subject will earn 0 euros.

- Examples:

- if $\bar{x}=1424$ and the guess is 1424 , the difference is 0 and earnings are 15 euros.

- if $\bar{x}=308$ and the guess is 311.5 , the difference is 3.5 and earnings are 11.5 euros.

- if $\bar{x}=803.25$ and the guess is 792 , the difference is 11.25 and earnings are 3.75 euros.

- se $\bar{x}=62.5$ and the guess is 30.5 , the difference is 32 and earnings are 0 euros, since $15-32<0$.

In each of this cases, the participant will also receive 5 euros for participating in the experiment.

- In each round, the optimal guess (which allows to get the maximum earnings) depends on the information that each subject has on the signals:

- if she knows only her own signal, the optimal choice is her own signal,

- if she knows or can deduce two signals, her optimal choice is the mean of the two signals;

- if she knows or can deduce three signals, her optimal choice is the mean of the three signals;

- if she knows or can deduce four signals, her optimal choice is the mean of the four signals.

\section{Information}

- In each of the tree phases 
- In the first round, each subject knows his own signal.

- From the second round onwards, before making his choice, each subject will be informed by the computer of the choices made in the previous rounds by some of the components of his group, based on the structure represented in the following figure:

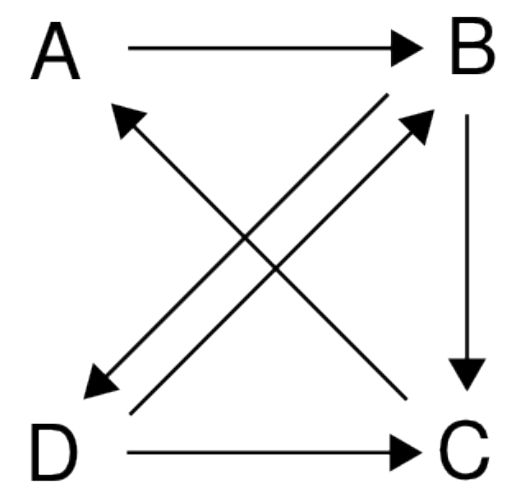

- Therefore, before making his choice

- A will be informed of the choices made by C.

- B will be informed of the choices made by D.

- $\mathrm{C}$ will be informed of the choices made by A and B.

- D will be informed of the choices made by A and C.

- The roles (A, B, C, D), the signals $\left(x_{A}, x_{B}, x_{C}, x_{D}\right)$ and by consequence their mean will change at each phase: the computer will generate them randomly before the first period of the phase.

\section{Feedback e payments}

- At the end of each phase the computer will show to each subject the four signals of his group, their mean, and the choices made.

- At the end of the experiment each subject will be shown the round the computer has selected to determine payments, the value of $\mathrm{x}$ for his group, the choice she made and the corresponding amount earned in euro.

- The experiment will terminate and the amount earned by each subject will be paid in cash.

\section{Control questions}


1. If you knew only your signal $(a)$, what would be your optimal guess?

2. If you knew your signal $(a)$ as well as the one of another member of your, group $(b)$, what would be your optimal guess?

3. If you knew your signal $(a)$ as well as the ones of two other members of your group $(b$ and $c$ ), what would be your optimal guess? ...............

4. If you knew your signal $(a)$ as well as the ones of three other members of your group $(b, c$, and $d)$, what would be your optimal guess? 


\section{References}

Acemoglu, D., K. Bimpikis, and A. Ozdaglar (2010). Dynamics of information exchange in endogenous social networks. Technical report, National Bureau of Economic Research.

Acemoglu, D., M. A. Dahleh, I. Lobel, and A. Ozdaglar (2011). Bayesian learning in social networks. The Review of Economic Studies 78(4), 12011236 .

Acemoglu, D. and A. Ozdaglar (2011). Opinion dynamics and learning in social networks. Dynamic Games and Applications 1(1), 3-49.

Bala, V. and S. Goyal (1998). Learning from neighbours. The Review of Economic Studies 65(3), 595-621.

Banerjee, A., A. G. Chandrasekhar, E. Duflo, and M. O. Jackson (2013). The diffusion of microfinance. Science 341(6144).

Banerjee, A. V. (1992). A simple model of herd behavior. The Quarterly Journal of Economics 107(3), 797-817.

Baños, R. A., J. Borge-Holthoefer, and Y. Moreno (2013). The role of hidden influentials in the diffusion of online information cascades. arXiv preprint arXiv:1303.4629.

Bikhchandani, S., D. Hirshleifer, and I. Welch (1992). A theory of fads, fashion, custom, and cultural change as informational cascades. Journal of political Economy 100(5), 992-1026.

Bonacich, P. (1972). Factoring and weighting approaches to status scores and clique identification. Journal of Mathematical Sociology 2(1), 113-120.

Buechel, B., T. Hellmann, S. Klößner, et al. (2012). Opinion dynamics under conformity. Institute of Mathematical Economics Working Paper (469).

Corazzini, L., F. Pavesi, B. Petrovich, and L. Stanca (2012). Influential listeners: An experiment on persuasion bias in social networks. European Economic Review 56(6), 1276-1288.

DeGroot, M. H. (1974). Reaching a consensus. Journal of the American Statistical Association 69(345), 118-121.

DeMarzo, P. M., D. Vayanos, and J. Zwiebel (2003). Persuasion bias, social influence, and unidimensional opinions. The Quarterly Journal of Economics 118(3), 909-968. 
Dittmer, J. C. (2001). Consensus formation under bounded confidence. Nonlinear Analysis-Theory Methods and Applications 47(7), 4615-4622.

Ellison, G. and D. Fudenberg (1993). Rules of thumb for social learning. Journal of Political Economy, 612-643.

Fischbacher, U. (2007). z-Tree: Zurich toolbox for ready-made economic experiments. Experimental economics 10(2), 171-178.

Fortunato, S. (2004). Universality of the Threshold for Complete Consensus for the Opinion Dynamics of Deffuant et al. International Journal of Modern Physics C 15(09), 1301-1307.

French, Jr, J. R. (1956). A formal theory of social power. Psychological review 63(3), 181.

Friedkin, N. E. and E. C. Johnsen (1990). Social influence and opinions. Journal of Mathematical Sociology 15(3-4), 193-206.

Gale, D. and S. Kariv (2003). Bayesian learning in social networks. Games and Economic Behavior 45(2), 329-346.

Gleeson, J. P., D. Cellai, J.-P. Onnela, M. A. Porter, and F. Reed-Tsochas (2013). A Simple Generative Model of Collective Online Behaviour. arXiv preprint arXiv:1305.7440.

Harary, F. (1959). A criterion for unanimity in French's theory of social power. In D. Cartwright (Ed.), Studies in social power. University of Michigan.

Hegselmann, R. and U. Krause (2002). Opinion dynamics and bounded confidence models, analysis, and simulation. Journal of Artificial Societies and Social Simulation 5(3).

Hegselmann, R. and U. Krause (2005). Opinion dynamics driven by various ways of averaging. Computational Economics 25(4), 381-405.

Jackson, M. O. (2010). Social and economic networks. Princeton University Press.

Jackson, M. O. and L. Yariv (2010). Diffusion, strategic interaction, and social structure. In J. Benhabib, A. Bisin, and M. O. Jackson (Eds.), Handbook of Social Economics. Elsevier.

Jadbabaie, A., P. Molavi, A. Sandroni, and A. Tahbaz-Salehi (2012). NonBayesian social learning. Games and Economic Behavior 76(1), 210-225. 
Jadbabaie, A., P. Molavi, and A. Tahbaz-Salehi (2013). Information Heterogeneity and the Speed of Learning in Social Networks. Working paper.

Katz, L. (1953). A new status index derived from sociometric analysis. Psychometrika 18(1), 39-43.

Möbius, M., T. Phan, and A. Szeidl (2010). Treasure hunt. Technical report, working paper.

Olfati-Saber, R. and R. M. Murray (2004). Consensus problems in networks of agents with switching topology and time-delays. Automatic Control, IEEE Transactions on 49(9), 1520-1533.

Perfect, H. and L. Mirsky (1965). The distribution of positive elements in doubly-stochastic matrices. Journal of the London Mathematical Society 1(1), 689-698.

Smith, L. and P. Sørensen (2000). Pathological outcomes of observational learning. Econometrica 68(2), 371-398. 